\title{
Rickets and Scurvy presenting in a Child as apparent Non Accidental Injury
}

\author{
D Lewis, C Carpenter, A Evans, P Thomas
}

\section{Citation}

D Lewis, C Carpenter, A Evans, $\mathrm{P}$ Thomas. Rickets and Scurvy presenting in a Child as apparent Non Accidental Injury. The Internet Journal of Orthopedic Surgery. 2006 Volume 4 Number 2.

\section{DOI: $10.5580 / 119 \mathrm{~d}$}

\begin{abstract}
The authors' report the case of an eleven month old Sudanese girl, who presented to the Accident and Emergency department with a femoral fracture. The fracture was apparently sustained following a minor, unsupervised fall. Subsequent radiographic imaging demonstrated changes which raised the concern of Non Accidental Injury. Only when a more detailed history was taken, and subsequent biochemical results became available was a diagnosis of nutritional rickets and scurvy made.
\end{abstract}

Following appropriate orthopaedic management, and dietary supplementation, the child made a full recovery. We highlight the importance of underlying biochemical abnormalities as a differential diagnosis in fractures presenting as Non Accidental Injury (NAI).

\section{CASE REPORT}

An eleven month old Sudanese girl was brought to the Accident and Emergency department by her parents, with a history of injury to her right thigh. The history as reported by the parents was that the child had fallen approximately $0.5 \mathrm{~m}$ from a bed onto a wooden floor, although the fall itself was not actually witnessed. The parents denied any other injuries, as well as any associated loss of consciousness.

On clinical examination the child was crying, and appeared distressed. The right mid thigh area was noted to be swollen, and the leg was externally rotated. X-Rays were taken of the right femur which showed a displaced, transverse fracture in the mid shaft region. The general appearance of the bones was felt to be abnormal, and evidence of periosteal reaction suggested the possibility of previous fractures, thus raising the concern of NAI (Fig I)

\section{Figure 1}

Figure 1: A plain radiograph of the right femur showing a displaced, transverse fracture through the mid shaft region. The underlying bone appears pathological, and there is evidence of periosteal new bone formation.

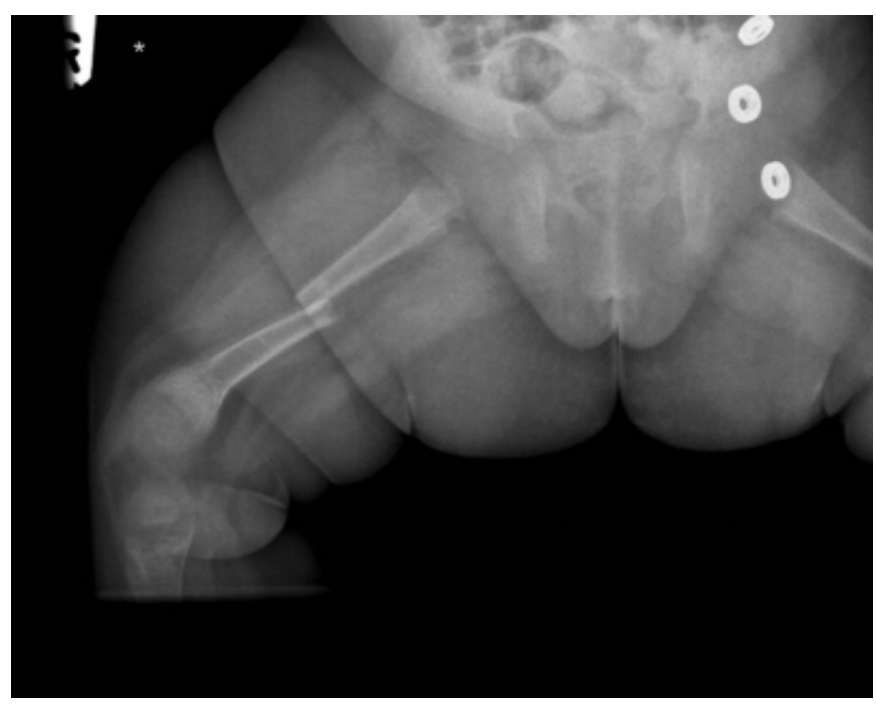

The child was subsequently placed in Gallows traction, a more detailed examination and history was conducted, and blood investigations were performed. At this stage child protection services were also involved due to the suspicion of NAI. 
On further examination the child appeared to be well nourished, and head circumference measurement was on the 98th centile for her age. The child was however noted to have a degree of frontal bossing, bilateral beading of the ribs, as well as swelling of both distal forearms in the metaphyseal areas. These changes were suggestive of rickets. This was confirmed on biochemical analysis with very low levels of 25-hydroxyl vitamin $\mathrm{D}$, in association with raised alkaline phosphatase, and parathyroid hormone. Also noted were low serum levels of ascorbic acid, suggesting a concurrent diagnosis of scurvy.

A skeletal survey of the child confirmed these diagnoses (Fig II), with classic radiographic changes seen. No further fractures were detected, and it was felt that the presenting injury was as a result of underlying pathological bone, and not NAI.

\section{Figure 2}

Figure 2: The whole of the skeleton is markedly demineralised, with extensive periosteal reaction along all of the long bones and ribs. The metaphyses are frayed, cupped and splayed. These appearances are those of gross rickets, with associated changes suggestive of concomitant scurvy.

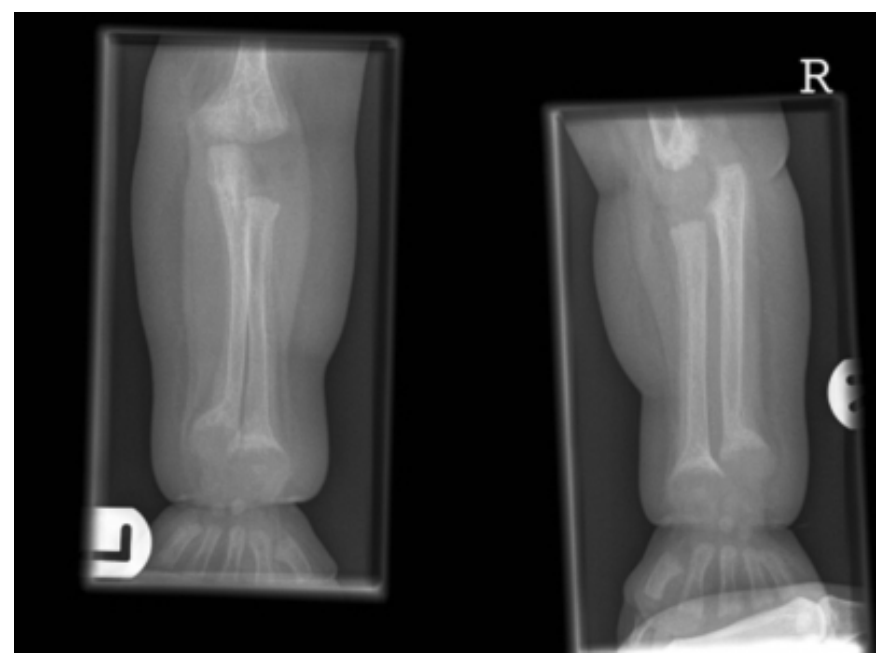

The mother explained that the child had been breast fed since birth, and had received little in the way of dietary supplements. These were started shortly after admission, and the fracture was treated non-operatively in a hip spice. The child was subsequently discharged home.

The child was reviewed regularly following discharge, and has since made a full recovery. Biochemical markers have returned to within normal limits and the child is now walking normally without any apparent adverse effects. Subsequent radiographic imaging has also shown marked resolution, following dietary supplementation of vitamins D and C. (Fig III)

\section{Figure 3}

Figure 3: A plain radiograph of the left wrist showing significant resolution of radiographic abnormalities following dietary supplementation.

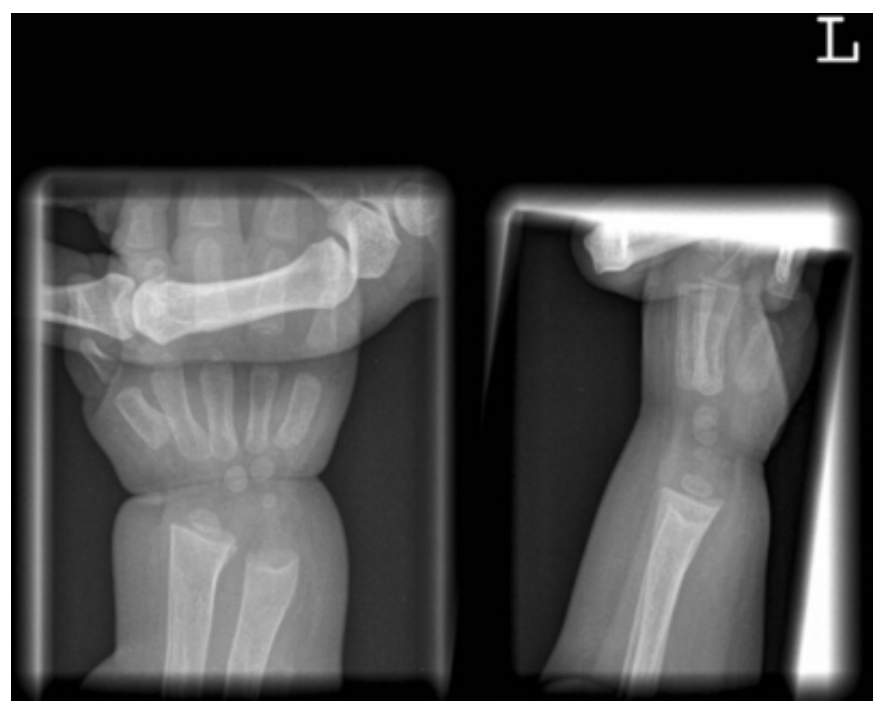

\section{DISCUSSION}

Rickets and scurvy are relatively rare conditions in western societies, but recent research has shown that they are presenting with increased frequency especially in ethnic minority groups. It has been suggested that this may be as a result of increase in asylum seeker numbers in certain areas. $\left({ }_{1}, 2,3\right)$. They may both result from dietary deficiencies, often as a result of prolonged breast feeding without nutritional supplementation. Reduced exposure to sunlight in these ethnic groups is also a recognised cause of rickets, as this is an important factor in vitamin D metabolism.

Our case demonstrates classical radiographic changes of both rickets and scurvy, with full resolution following appropriate treatment. We highlight the fact that radiographic examination of one bone in isolation may be confused with Non Accidental Injury. It is in the metaphyseal area of bone that nutritional deficiencies cause the most marked radiographic characteristics, and therefore it is important to adequately image the joint above and below the site of fracture.

We highlight the importance of ruling out underlying pathological processes of bone that cause a predisposition to fracture, when investigating potential causes of Non Accidental Injury.

\section{CORRESPONDENCE TO}

Miss E C Carpenter Department of Trauma and Orthopaedic 
Surgery. University Hospital of Wales, Heath Park, Cardiff. CF14 4XN Tel No: 02920748044 Fax No: 02920744206 Email: carpie@doctors.org.uk

\section{References}

1. Shaw NJ, Pal BR. Vitamin D deficiency in UK Asian families: activating a new concern. Arch Dis Child 2002; 86: 147-9

2. S Ladhani, L Srinivasan, C Buchanan, J Allgrove.

Presentation of vitamin D deficiency. Arch Dis Child 2004; 89: 781-784

3. T R Welch. Vitamin D-deficiency rickets: The reemergence of a once conquered disease. J Paediatrics 2000; 137: $143-5$. 


\section{Author Information}

Dan Lewis, MRCS

Specialist Registrar in Trauma and Orthopaedic Surgery, University Hospital of Wales

\section{Clare Carpenter, MRCS}

Specialist Registrar in Trauma and Orthopaedic Surgery, University Hospital of Wales

\section{Aled Evans, FRCS(Orth)}

Specialist Registrar in Trauma and Orthopaedic Surgery, University Hospital of Wales

Phill Thomas, FRCS(Orth)

Consultant in Trauma and Orthopaedic Surgery, University Hospital of Wales 